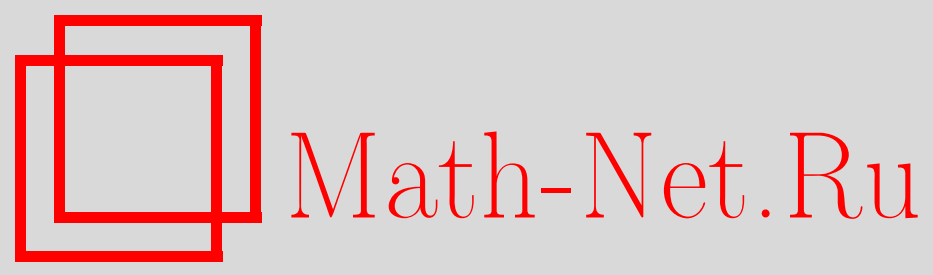

Ю. Г. Прохоров, Ограниченность исключительных факторособенностей, Матем. заметки, 2000, том 68, выпуск $5,786-789$

DOI: https://doi.org/10.4213/mzm999

Использование Общероссийского математического портала Math-Net.Ru подразумевает, что вы прочитали и согласны с пользовательским соглашением http://www.mathnet.ru/rus/agreement

Параметры загрузки:

IP : 54.237 .59 .107

26 апреля 2023 г., 14:08:18

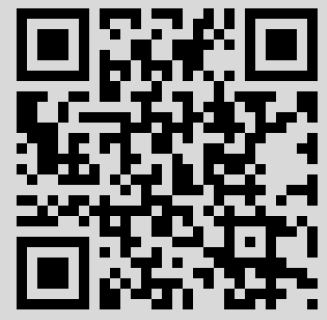




\section{ОГРАНИЧЕННОСТЬ ИСКЛЮЧИТЕЛЬНЫХ ФАКТОРОСОБЕННОСТЕЙ}

\section{Ю.Г. Прохоров}

1. Введение. Настоящая заметка является продолжением работы [1] и посвящена изучению исключительных факторособенностей. Всюду на протяжении заметки основное поле предполагается полем $\mathbb{C}$. Мы будем пользоваться основными понятиями лог-программы минимальных моделей (см. [2]).

ОПРЕДЕЛЕНИЕ 1.1 (см. $[3,5.6])$. Пусть $(X \ni P)$-нормальная особенность и пусть $D=\sum d_{i} D_{i}$ - эффективный $\mathbb{Q}$-дивизор на $X$ такой, что пара $(X, D)$ имеет лишь логканонические особенности. Говорят, что пара $(X, D)$ исключительн $а$, если существует не более одного дивизора $E$ поля функций $\mathscr{K}(X)$ с дискрепантностью $a(E, D)=-1$. Логканоническая особенность $(X \ni P)$ называется исключительной, если пара $(X, D)$ исключительна всякий раз, когда она логканонична.

Мы рассмотрим с точки зрения исключительности один специальный класс особенностей - факторособенности по конечным группам. Эти особенности логтерминальны $[2,20.3 .3]$. Согласно хорошо известной теореме Шефарда-Тодда-Шевалле (см., например, $[4,4.2 .5])$ при изучении факторособенностей мы без ограничения общности можем ограничиться группами $G \subset G L_{n}(\mathbb{C})$, не содержащими отражений (т.е. элементов $g$ таких, что $\mathrm{rk}(g-\mathrm{id})=1)$. Основной результат настоящей заметки - следующая теорема.

Теорема 1.1. Зафиксируем $\varepsilon>0$. Тогда мнохество конечных подгрупп $G \subset G L_{n}(\mathbb{C})$, не содержащ,их отражсний и таких, что $\mathbb{C}^{n} / G$ - исключительная $\varepsilon$-логтерминальная особенность, является конечным с точностью до сопряжений.

СледствиЕ 1.1. Множество конечных подгрупп $G \subset G L_{n}(\mathbb{C})$, не содержащих отражсений и таких, что $\mathbb{C}^{n} / G$ - исключительная каноническая особенность, является конечным с точностью до сопряжсений.

Аналогичный результат для гиперповерхностных особенностей получен в [5].

2. Исключительные группы примитивны. В настоящем пункте мы докажем теорему 1.1. Утверждение является следствием предложения 2.1 и классической теоремы Жордана (см. теорему 2.1).

Обозначения. Положим $V:=\mathbb{C}^{n}$. Пусть $G \subset G L_{n}(\mathbb{C})$ - конечная подгруппа, не содержащая отражений, и пусть $\pi: V \rightarrow X=V / G$ - морфизм факторизации. Пусть $\sigma: \widetilde{V} \rightarrow V$ - раздутие точки 0 и пусть $E:=\sigma^{-1}(0)$ - исключительный дивизор. Рассмотрим коммутативную диаграмму

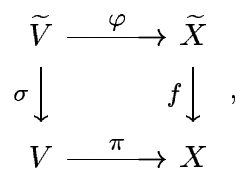

где $\widetilde{X}:=X / G$, и положим $S:=\varphi(E)$.

Лемма 2.1. В принятых обозначениях пусть $B$ - эффективный $\mathbb{Q}$-дивизор на $X$ такой, что пара $(X, B)$ логканонична, но не логтерминальна по Kавамате, и пусть $B^{\prime}:=\pi^{*} B$. Предполохсим, что особенность $(X \ni$ э) исключительна. Тогда дискрепантность (см. $[2$, гл. 2]) дивизора $E$ относительно $B^{\prime}$ равна $a\left(E, B^{\prime}\right)=-1$.

Работа выполнена при частичной поддержке INTAS-OPEN, грант № 97-2072, Российского фонда фундаментальных исследований, грант № 99-01-01132, и программы ПВНШ, грант № 96-1596146. 
ДокАЗАТЕльство. По $[2,20.3]$ пара $\left(V, B^{\prime}\right)$ логканонична, но не логтерминальна по Кавамате. Так как особенность ( $X \ni o$ ) исключительна, $a(S, B)=-1$ (см. $[1,2.7])$. Следовательно, $a\left(E, B^{\prime}\right)=-1($ см. $[2$, доказательство 20.3]).

ОПРЕдЕЛЕниЕ 2.1. Пусть $G \subset G L_{n}(\mathbb{C})$ - конечная неприводимая подгруппа. Она называется импримитивной, если существует нетривиальное разложение $\mathbb{C}^{n}=\bigoplus_{i} V_{i}$ такое, что $g \cdot V_{i}=V_{j}$ для всех $g \in G$. В противном случае говорят, что $G$ примитивна.

ПРЕДЛОЖЕнИЕ 2.1 (ср. $[1,3.4])$. Пусть $G \subset G L_{n}(\mathbb{C})$ - конечная подгруппа, не содержащ,ая отражений. Предположим, что особенность $\mathbb{C}^{n} / G$ исключительна. Тогда группа $G$ примитивна.

ДокАЗАТЕЛЬСтво. Мы будем придерживаться принятых обозначений. Согласно $[1,3.6]$ представление $G \hookrightarrow G L_{n}(\mathbb{C})$ неприводимо. Предположим, что группа $G$ импримитивна. Пусть $V=$ $\bigoplus_{j=1}^{m} V_{j}$ - разложение из определения 2.1 и пусть $G_{0} \subset G$ - максимальная нормальная подгруппа такая, что $G_{0} \cdot V_{j}=V_{j}$ для всех $j=1, \ldots, m$. Пусть $H_{1}$ - гиперплоское сечение в $\mathbb{C}^{n}$, содержащее $V_{2} \oplus \cdots \oplus V_{m}$, и пусть $\left\{H_{1}, \ldots, H_{r}\right\}$ - его $G$-орбита. Положим

$$
D:=\frac{n}{r} \sum_{i=1}^{r} H_{i}
$$

Мы покажем, что пара $\left(\mathbb{C}^{n}, D\right)$ логканонична и неисключительна. Имеем

$$
a(E, D)=a(E, 0)-\operatorname{mult}_{E}(D)=n-1-\frac{n}{r} \sum_{i=1}^{r} \operatorname{mult}_{E}\left(H_{i}\right)=-1 .
$$

Если пара $\left(\mathbb{C}^{n}, D\right)$ не логканонична, то пара $\left(\mathbb{C}^{n}, \alpha D\right)$ логканонична, но не логтерминальна по Кавамате для некоторого $0<\alpha<1$. Поэтому $a(E, \alpha D)>-1$. Ясно что, $\alpha D=\pi^{*} B$ для некоторого $\mathbb{Q}$-дивизора $B$ на $X$. По лемме 2.1 имеем $a(E, \alpha D)=-1$; противоречие.

Далее, пусть $\sigma_{1}: X_{1} \rightarrow V$ раздутие $V_{1}$ и пусть $E_{1}-$ исключительньй дивизор. Достаточно показать, что $a\left(E_{1}, D\right)=-1$. Действительно,

$$
\begin{aligned}
a\left(E_{1}, D\right) & =a\left(E_{1}, 0\right)-\operatorname{mult}_{E_{1}} D=n-\operatorname{dim} V_{1}-1-\frac{n}{r} \sum_{H_{i} \supset V_{1}} \operatorname{mult}_{E_{1}}\left(H_{i}\right) \\
& =n-\frac{n}{m}-1-\frac{n}{r}\left(\operatorname{Card}\left\{i \mid H_{i} \supset V_{1}\right\}\right)=n-\frac{n}{m}-1-\frac{n(m-1)}{m}=-1,
\end{aligned}
$$

где

$$
\operatorname{Card}\left\{i \mid H_{i} \supset V_{1}\right\}=\frac{1}{m} \operatorname{Card}\left\{(i, j) \mid H_{i} \supset V_{j}\right\}=\frac{r}{m} \operatorname{Card}\left\{j \mid H_{1} \supset V_{j}\right\}=\frac{r(m-1)}{m}
$$

Таким образом, мы построили логканоническую границу $D$ на $V$ и, по крайней мере, два дивизоpa $E, E_{1}$ с дискрепантностями, что противоречит исключительности. Предложение доказано.

Следствие 2.1. В условиях предложения 2.1 любая нормальная абелева әруппа $A \subset G$ содерхится в чентре $G$.

ДокАЗАТЕЛЬСТво. Если $\mathbb{C}^{n}=\bigoplus_{i} V_{i}$ - разложение в сумму максимальных собственных подпространств для группы $A$, то оно является импримитивным разложением для $G$.

Согласно следующей классической теореме число примитивных групп в каждой фиксированной размерности ограничено с точностью до сопряжений и добавлений скалярных матриц. 
Теорема 2.1 (Жордан [6]). Существует функция $J: \mathbb{N} \rightarrow \mathbb{N}$ такая, что всякая конечная подәруппа $G \subset G L_{n}(\mathbb{C})$ обладает нормальной абелевой подгруппой, индекс которой не превосходит $J(n)$.

Таким образом, для того чтобы закончить доказательство теоремы 1.1, мы должны показать, что порядок $N$ центра $Z(G)$ группы $G$ ограничен константой, зависящей от $\varepsilon$ и $n$. Это может быть сделано с использованием аргументов [1, доказательство 3.15]. Для удобства читателя мы воспроизведем эти соображения. Разложим морфизм $\pi$ как $\pi: V \stackrel{\pi_{1}}{\rightarrow} X^{\prime \prime} \stackrel{\pi_{2}}{\rightarrow} X$, где $X^{\prime \prime}:=V / Z(G)$. Тогда $-1+\varepsilon<\operatorname{discr}(X) \leqslant \operatorname{discr}\left(X^{\prime \prime}\right)$ (см., например, [2, 20.3]). Торическая особенность $X^{\prime \prime}$ может быть разрешена при помощи единственного раздутия, задающего исключительньй дивизор с дискрепантностью $-1+n / N$. Следовательно, $-1+\varepsilon<-1+n / N$. Таким образом, $N<n / \varepsilon$, что и доказывает теорему.

3. Следствия. Следующее утверждение дает дополнительные ограничения на группы, факторы по которым - исключительные особенности.

ПРЕДЛОЖЕНИЕ 3.1 (cp. [1]). Пусть $G \subset G L_{n}(\mathbb{C})$ - малая конечная подгруппа такая, что факторособенность $\mathbb{C}^{n} / G$ исключительна. Тогда $G$ не имеет полуинвариантов степе$\psi u \leqslant n$.

Напомним, что многочлен $\zeta \in \mathbb{C}\left[x_{1}, \ldots, x_{n}\right]$ называется полуинвариантом группы $G \subset$ $G L_{n}(\mathbb{C})$, если существует характер $\chi: G \rightarrow \mathbb{C}^{*}$ такой, что $g \cdot \zeta=\chi(g) \zeta$ для всех $g \in G$.

ДоКАЗАТЕЛЬСТвО. Используются принятые в п. 2 обозначения. Предположим, что $G$ имеет однородныи инвариант $\zeta$ степени $d \leqslant n$. Положим $F:=\left\{x \in \mathbb{C}^{n} \mid \zeta(x)=0\right\}$. Возьмем $\alpha \in \mathbb{Q}$ такой, что $K_{V}+\alpha F$ максимально логканоничен. Согласно $[1,2.6]$ достаточно показать, что $\alpha=1$. Предположим, что $\alpha<1$. Имеем

$$
K_{\widetilde{V}}=\sigma^{*} K_{V}+(n-1) E, \quad \bar{F}=\sigma^{*} F-d F,
$$

где $d=\operatorname{deg} F=\operatorname{mult}_{0}(F) \leqslant n$. Это дает нам

$$
\sigma^{*}\left(K_{V}+\alpha F\right)=K_{\tilde{V}}+\alpha \bar{F}+(\alpha d+1-n) E .
$$

Следовательно, $a(E, \alpha F)=-(\alpha d+1-n)>-1$. С другой стороны, по лемме $2.1 a(E, \alpha F)=-1$, противоречие.

ЗАмЕч АниЕ 3.1. В [1] было доказано, что обратная импликация имеет место при $n \leqslant 3$. Пример ниже показывает что это неверно в высших размерностях.

ПримеР 3.1. Пусть $\Gamma \subset S L_{2}(\mathbb{C})$ - бинарная группа икосаэдра. Рассмотрим подгруппу

$$
G=\left\{\left(\begin{array}{cc}
A & 0 \\
0 & B
\end{array}\right) \mid A, B \in \Gamma\right\} \subset S L_{4}(\mathbb{C})
$$

Тогда особенность $\mathbb{C}^{4} / G$ неисключительна (поскольку представление $G \hookrightarrow S L_{4}(\mathbb{C})$ приводимо $[1$, 3.6]). С другой стороны, кольцо инвариантов представляется в виде

$$
\mathbb{C}\left[x_{1}, x_{2}, y_{1}, y_{2}\right]^{G} \simeq \mathbb{C}\left[x_{1}, x_{2}\right]^{\Gamma} \otimes \mathbb{C}\left[y_{1}, y_{2}\right]^{\Gamma} .
$$

Так как $\Gamma$ не имеет полуинвариантов степени $\leqslant 4$ (см. $[4,4.5 .5])$, это же верно и для $G$.

В заключение мы покажем, что при каждом фиксированном $n$ существует лишь конечное число типов исключительных особенностей $\mathbb{C}^{n} / G$.

ЛЕмма 3.1. Пусть $G, G^{\prime} \subset G L_{n}(\mathbb{C})$ - конечные подгруппь, не содержащие отрахсний и такие, что их образы в $P G L_{n}(\mathbb{C})$ совпадают. Тогда исключительность особенностей $\mathbb{C}^{n} / G$ и $\mathbb{C}^{n} / G^{\prime}$ әквивалентна. 
ДокАЗАТЕльство. В обозначениях п. 2 имеем $K_{\tilde{V}}+E=\varphi^{*}\left(K_{\tilde{X}}+S\right)$. Следовательно, пара $(\widetilde{X}, S)$ чисто логтерминальна $[2,20.3]$. В [7] было доказано, что исключительность $\mathbb{C}^{n} / G$ определяется парой $\left(S, \operatorname{Diff}_{S}\right)$, причем в нашей ситуаци и $\operatorname{Diff}_{S}$ находится из формулы ветвления $\left.\varphi\right|_{E} ^{*}\left(K_{S}+\operatorname{Diff}_{S}\right)=K_{E}$. Таким образом, пара $\left(S, \operatorname{Diff}_{S}\right)$ полностью задается действием группы $G /\left(G \cap \mathbb{C}^{*}\right)$ на $E=\mathbb{P}(V)$ и не зависит от добавления скалярных матриц.

Используя лемму 3.1 , из предложения 2.1 и теоремы 2.1 получаем.

СледствиЕ 3.1. Множество конечных подгрупп $G \subset G L_{n}(\mathbb{C})$, не содержащих отражений и таких, что $\mathbb{C}^{n} / G$ - исключительная особенность, является конечным $с$ точностью до сопря⿻ений и скалярных умножсений.

Исключительные факторособенности в размерности $n \leqslant 3$ классифицированы [1]. При $n \geqslant 4$ вопрос остается открытьм.

\section{СПИСОК ЦИТИРОВАННОЙ ЛИТЕРАТУРЫ}

1. Markushevich D., Prokhorov Yu. G. // Amer. J. Math. 1999. V. 121. №6. P. 1179-1189. 2. Kollár J. Flips and abundance for algebraic threefolds // Summer Seminar at the University of Utah (Salt Lake City, 1991). Astérisque. V. 211. Paris, 1992. 3. Шокуров В. В. // Изв. АН СССР. Сер. матем. 1992. Т. 56. № 1. С. 105-203. 4. Спрингер Т. Теория инвариантов. М.: Мир, 1981. 5. Ishii S., Prokhorov Yu. G. Hypersurface exceptional singularities // http://xxx.lanl.gov/ abs/math/9910123, 1999. 6. Jordan C. Traité des substitutions des equations algebriques. Paris, 1870. 7. Prokhorov Yu. G. // Proc. of Conf. in Memory of A. G. Kurosh. Berlin: de Gruyter, 2000. P. 301-317.

Московский государственный университет им. М.В. Ломоносова 